Pacific Journal of Mathematic 


\title{
ON THE IRRATIONALITY OF CERTAIN SERIES
}

\author{
P. Erdös and E. G. Straus
}

A criterion is established for the rationality of series of the form $\sum b_{n} /\left(a_{1}, \cdots, a_{n}\right)$ where $a_{n}, b_{n}$ are integers, $a_{n} \geqq 2$ and $\lim b_{n} /\left(a_{n-1} a_{n}\right)=0$. This criterion is applied to prove irrationality and rational independence of certain special series of the above type.

1. Introduction. In an earlier paper [2] we proved the following result:

THEOREM 1.1. If $\left\{a_{n}\right\}$ is a monotonic sequence of positive integers with $a_{n} \geqq n^{11 / 12}$ for all large $n$, then the series

$$
\sum_{n=1}^{\infty} \frac{\varphi(n)}{a_{1} a_{2} \cdots a_{n}} \text { and } \sum_{k=1}^{\infty} \frac{\sigma(n)}{a_{1} a_{2} \cdots a_{n}}
$$

are irrational.

We conjectured that the series (1.2) are irrational under the single assumption that $\left\{a_{n}\right\}$ is monotonic and we observed that some such condition is needed in view of the possible choices $a_{n}=\varphi(n)+1$ or $a_{n}=\sigma(n)+1$. These particular choices do not satisfy the hypothesis $\lim \inf a_{n+1} / a_{n}>0$ but we do not know whether that hypothesis which is weaker than that of the monotonicity of $a_{n}$ would suffice.

In this note we obtain various improvements and generalizations of Theorem 1.1, in particular by relaxing the growth conditions on the $a_{n}$ and using more precise results in the distribution of primes.

In $\S 2$ we obtain some general conditions for the rationality of series of the form $\sum b_{n} /\left(a_{1}, \cdots, a_{n}\right)$ which are modifications of [2, Lemma 2.29]. In $\S 3$ we use a result of A. Selberg [3] on the regularity of primes in intervals to obtain improvements and generalizations of Theorem 1.1.

2. Criteria for rationality.

THEOREM 2.1. Let $\left\{b_{n}\right\}$ be a sequence of integers and $\left\{a_{n}\right\} a$ sequence of positive integers with $a_{n}>1$ for all large $n$ and

$$
\lim _{n=1} \frac{\left|b_{n}\right|}{a_{n-1} a_{n}}=0 \text {. }
$$

Then the series 


$$
\sum_{n=1}^{\infty} \frac{b_{n}}{a_{1} \cdots a_{n}}
$$

is rational if and only if there exists a positive integer $B$ and $a$ sequence of integers $\left\{c_{n}\right\}$ so that for all large $n$ we have

$$
B b_{n}=c_{n} a_{n}-c_{n+1}, \quad\left|c_{n+1}\right|<a_{n} / 2 .
$$

Proof. Assume that (2.4) holds beyond N. Then

$$
\begin{aligned}
B a_{1} \cdots a_{N-1} \sum_{n=1}^{\infty} \frac{b_{n}}{a_{1} \cdots a_{n}} & =\text { integer }+\sum_{n=N}^{\infty} \frac{c_{n} a_{n}-c_{n+1}}{a_{N} \cdots a_{n}} \\
& =\text { integer }+c_{N}=\text { integer } .
\end{aligned}
$$

Thus condition (2.4) is sufficient for the rationality of the series (2.3).

To prove the necessity of (2.4) assume that the series (2.3) equals $A / B$ and that $N$ is so large that $a_{n} \geqq 2$ and $\left|b_{n} /\left(a_{n-1} a_{n}\right)\right|<1 /(4 B)$ for all $n \geqq N$. Then

$$
\begin{aligned}
A a_{1} \cdots a_{N-1} & =B a_{1} \cdots a_{N-1} \sum_{n=1}^{\infty} \frac{b_{n}}{a_{1} \cdots a_{n}} \\
& =\text { integer }+\frac{B b_{N}}{a_{N}}+\sum_{n=N+1}^{\infty} \frac{B b_{n}}{a_{N} \cdots a_{n}} .
\end{aligned}
$$

If we call the last sum $R_{N}$ we get

$$
\begin{aligned}
\left|R_{N}\right| & \leqq \max _{n>N} \frac{\left|B b_{n}\right|}{a_{n-1} a_{n}} \sum_{n=N+1}^{\infty} \frac{1}{a_{N} \cdots a_{n-2}} \\
& <\frac{1}{4} \sum_{k=0}^{\infty} \frac{1}{2^{k}}=\frac{1}{2} .
\end{aligned}
$$

Thus, if we choose $c_{N}$ to be the integer nearest to $B b_{N} / a_{N}$ and write $B b_{N}=c_{N} a_{N}-c_{N+1}$ then (2.5) yields that $-c_{N+1} / a_{N}+R_{N}$ is an integer of absolute value less than 1 and hence 0 , so that

$$
\frac{c_{N+1}}{a_{N}}=R_{N}=\frac{B b_{N+1}}{a_{N} a_{N+1}}+\frac{1}{a_{N}} R_{N+1}
$$

or

$$
\frac{B b_{N+1}}{a_{N+1}}=c_{N+1}-R_{N+1} .
$$

From (2.8) it follows that $c_{N+1}$ is the integer nearest to $B b_{N+1} / a_{N+1}$ and if we write $B b_{N+1}=c_{N+1} a_{N+1}-c_{N+2}$ we get

$$
\frac{B b_{N+2}}{a_{N+2}}=c_{N+2}-R_{N+2} \text {. }
$$


Proceeding in this manner we get the desired sequence $\left\{c_{n}\right\}$.

REMARK. Since (2.2) implies $R_{n} \rightarrow 0$ it follows that for rational values of the series (2.3) we get $c_{n+1} / a_{n} \rightarrow 0$. Thus either $a_{n} \rightarrow \infty$ or $c_{n}=0$ and hence $b_{n}=0$ for all large $n$.

COROLLARY 2.10. Let $\left\{a_{n}\right\},\left\{b_{n}\right\}$ satisfy the hypotheses of Theorem 2.1 and in addition the conditions that for all large $n$ we have $b_{n}>0, a_{n+1} \geqq a_{n}, \lim \left(b_{n+1}-b_{n}\right) / a_{n} \leqq 0$ and $\lim \inf a_{n} / b_{n}=0$. Then the series (2.3) is irrational.

Proof. According to Theorem 2.1 the rationality of (2.3) implies the existence of a positive integer $B$ and a sequence of integers $\left\{c_{n}\right\}$ so that

$$
B b_{n}=c_{n} a_{n}-a_{n+1}
$$

for all large $n$ where $c_{n+1} / a_{n} \rightarrow 0$. Thus

$$
\frac{b_{n+1}}{b_{n}}=\frac{c_{n+1} a_{n+1}-c_{n+2}}{c_{n} a_{n}-c_{n+1}}>\frac{\left(c_{n+1}-\varepsilon\right)}{c_{n} a_{n}} \geqq \frac{c_{n+1}-\varepsilon}{c_{n}}
$$

for all $\varepsilon>0$ and sufficiently large $n$. Thus $c_{n+1}>c_{n}$ would lead to

$$
\begin{aligned}
b_{n+1} & >\left(1+\frac{1-\varepsilon}{c_{n}}\right) b_{n}>b_{n}+(1-\varepsilon)\left(a_{n}-\frac{c_{n+1}}{c_{n}}\right) / B \\
& >b_{n}+(1-\varepsilon)^{2} a_{n} / B .
\end{aligned}
$$

This contradicts our hypothesis for sufficiently large $n$. Thus we get $0<c_{n+1} \leqq c_{n}$ for all large $n$ and hence $b_{n} / a_{n}$ is bounded contrary to the hypothesis that $\lim \inf a_{n} / b_{n}=0$.

In fact, if we omit the hypothesis $\lim \inf a_{n} / b_{n}=0$ then we get rational values for the series (2.3) only when $B b_{n}=C\left(a_{n}-1\right)$ with positive integers $B, C$ for all large $n$.

3. Some special sequences.

THEOREM 3.1. Let $p_{n}$ be the $n$th prime and let $\left\{a_{n}\right\}$ be a monotonic sequence of positive integers satisfying $\lim p_{n} / a_{n}^{2}=0$ and $\lim \inf a_{n} / p_{n}=$ 0. Then the series

$$
\sum_{n=1}^{\infty} \frac{p_{n}}{a_{1} \cdots a_{n}}
$$

is irrational.

Proof. Since the series (3.2) satisfies the hypotheses of Theorem 
2.1 it follows that there is a sequence $\left\{c_{n}\right\}$ and an integers $B$ so that for all large $n$ we have

$$
B p_{n}=c_{n} a_{n}-c_{n+1} .
$$

For large $n$ an equality $c_{n}=c_{n+1}$ would imply $c_{n} \mid B$ and $a_{n}>p_{n}$. Since $\left\{c_{n}\right\}$ is unbounded there must exist an index $m \geqq n$ so that $c_{m} \leqq c_{n}<c_{m+1}$. But this implies by an argument analogous to (2.11) that

$$
p_{m+1}>p_{m}+a_{m} /(2 B)>\left(1+\frac{1}{2 B}\right) p_{m}
$$

which is impossible for large $m$. Thus we may assume that $c_{n} \neq c_{n+1}$ for all large $n$. Now consider an interval $N \leqq n \leqq 2 N$. If $c_{n+1}>c_{n}$ then as in (3.4) we get

$$
p_{n+1}>p_{n}+a_{n} /(2 B)>p_{n}+\sqrt{p_{n}}
$$

which therefore happens for fewer than $\left(p_{2 N}-p_{N}\right) / \sqrt{p_{N}}<N^{1 / 2+\varepsilon}$ values in the interval $(N, 2 N)$. If $c_{n+1}<c_{n}$ then we get

$$
1>\frac{c_{n} a_{n}-c_{n+1}}{c_{n+1} a_{n+1}-c_{n+2}}>\frac{c_{n}\left(a_{n}-1\right)}{c_{n+1} a_{n+1}}>\left(1+\frac{1}{c_{n+1}}\right) \frac{a_{n}-1}{a_{n+1}}
$$

so that

$$
a_{n+1}>a_{n}+\frac{a_{n}-1}{c_{n+1}}>a_{n}+1 .
$$

Since case (3.5) holds for more than $N / 2$ values of $n$ in $(N, 2 N)$ we get $a_{2 N}>N / 2$ and thus for all large $n$ we have $a_{n}>n / 4, c_{n}<$ $p_{n} / a_{n}+1<\sqrt{n} / 4$. Substituting these values in (3.5) we get

$$
a_{n+1}>a_{n}+\sqrt{n} \text { when } c_{n+1}<c_{n}, n \text { large ; }
$$

so that $a_{2 N}>N^{3 / 2} / 2$, contradicting the hypothesis that $\lim \inf a_{n} / p_{n}=0$.

THEOREM 3.7. Let $\left\{a_{n}\right\}$ be a monotonic sequence of positive integers with $a_{n}>n^{1 / 2+\delta}$ for some positive $\delta>0$ and all large $n$. Then the numbers $1, x, y, z$ are rationally independent. Here

$$
x=\sum_{n=1}^{\infty} \frac{\varphi(n)}{a_{1} \cdots a_{n}}, \quad y=\sum_{n=1}^{\infty} \frac{\sigma(n)}{a_{1} \cdots a_{n}}
$$

and

$$
z=\sum_{n=1}^{\infty} \frac{d_{n}}{a_{1} \cdots a_{n}}
$$


where $\left\{d_{n}\right\}$ is any sequence of integers satisfying $\left|d_{n}\right|<n^{1 / 2-\delta}$ for all large $n$ and infinitely many $d_{n} \neq 0$.

Proof. Assume that there exist integers $A, B, C$ not all 0 so that setting $b_{n}=A \varphi(n)+B \sigma(n)+C d_{n}$ we get that $S=\sum_{n=1}^{\infty} b_{n} /\left(a_{1}, \cdots, a_{n}\right)$ is an integer.

From Theorem 2.1 it follows directly that $z$ is irrational and thus not both $A$ and $B$ can be zero. We consider first the case $A+B \neq 0$ so that without loss of generality we may assume $A+B=D>0$. Since $S$ satisfies the hypotheses of Theorem 2.1 there exist integers $\left\{c_{n}\right\}$ so that

$$
b_{n}=c_{n} a_{n}-c_{n+1} \text { for all large } n .
$$

Since $\left|b_{n}\right|<n^{1+\delta / 2}$ for all large $n$ we get

$$
\left|c_{n}\right|<n^{(1-\hat{)}) / 2} \text { for all large } n \text {. }
$$

Let $p_{n}$ be the $n$th prime and set

$$
a_{n}^{\prime}=a_{p_{n}}, b_{n}^{\prime}=b_{p_{n}}, c_{n}^{\prime}=c_{p_{n}}, c_{n}^{\prime \prime}=c_{p_{n}+1},
$$

then

$$
b_{n}^{\prime}=A\left(p_{n}-1\right)+B\left(p_{n}+1\right)+C d_{p_{n}}=D_{p_{n}}+d_{n}^{\prime}
$$

where

$$
d_{n}^{\prime}=C d_{p_{n}}-A+B \text { with }\left|d_{n}^{\prime}\right|<n^{(1-\delta) / 2} \text { for all large } n .
$$

Now

$$
\begin{aligned}
b_{n}^{\prime} & =c_{n}^{\prime} a_{n}^{\prime}-c_{n}^{\prime \prime} \\
b_{n+1}^{\prime} & =c_{n+1}^{\prime} a_{n+1}^{\prime}-c_{n+1}^{\prime \prime}
\end{aligned}
$$

so that from

$$
\begin{aligned}
\frac{b_{n+1}^{\prime}}{b_{n}^{\prime}} & =\frac{D p_{n+1}+d_{n+1}^{\prime}}{D p_{n}+d_{n}^{\prime}}=\frac{p_{n+1}}{p_{n}} \frac{1+d_{n+1}^{\prime} /\left(D p_{n+1}\right)}{1+d_{n}^{\prime} /\left(D p_{n}\right)} \\
& =\frac{p_{n+1}}{p_{n}}\left(1+o\left(n^{-(1+\delta) / 2}\right)\right)
\end{aligned}
$$

we get

$$
\begin{aligned}
\frac{p_{n+1}}{p_{n}} & =\frac{c_{n+1}^{\prime} a_{n+1}^{\prime}-c_{n+1}^{\prime \prime}}{c_{n}^{\prime} a_{n}^{\prime}-c_{n}^{\prime \prime}}\left(1+o\left(n^{-(1+\delta) / 2}\right)\right) \\
& =\frac{c_{n+1}^{\prime}}{c_{n}^{\prime}} \frac{1-c_{n+1}^{\prime \prime} /\left(a_{n+1}^{\prime} c_{n+1}^{\prime}\right)}{1-c_{n}^{\prime \prime} /\left(a_{n}^{\prime} c_{n}^{\prime}\right)}\left(1+o\left(n^{-(1+\delta) / 2}\right)\right) \\
& =\frac{c_{n+1}^{\prime}}{c_{n}^{\prime}}\left(1+o\left(n^{-(1+\delta) / 2}\right)\right) .
\end{aligned}
$$


Here the last inequality follows from the fact that

$$
\begin{aligned}
\left|\frac{c_{n+1}}{c_{n}}\right| & =\left|\frac{\left(b_{n+1}+c_{n+2}\right) / a_{n+1}}{\left(b_{n}+c_{n+1}\right) / a_{n}}\right|=\frac{|A \varphi(n+1)+B \sigma(n+1)|+O\left(n^{(1-\delta) / 2}\right)}{|A \varphi(n)+B \sigma(n)|+O\left(n^{(1-\delta) / 2}\right)} \\
& =o\left(n^{\delta / 2}\right) .
\end{aligned}
$$

From (3.8) we get that $c_{n+1}^{\prime}>c_{n}^{\prime}$ implies

$$
p_{n+1}>p_{n}+\frac{p_{n}}{c_{n}^{\prime}}-p_{n}^{1 / 2-\delta / 4}>p_{n}+\frac{1}{2} p_{n}^{1 / 2+\check{\delta}}
$$

for all large $n$.

We now use the following result of A. Selberg [3, Theorem 4].

THEOREM 3.10. Let $\Phi(x)$ be positive and increasing and $\Phi(x) / x$ decreasing for $x>0$, further suppose

$\Phi(x) / x \rightarrow 0$ and $\quad \lim \inf \log \Phi(x) / \log x>19 / 77$ for $\quad x \rightarrow \infty$.

Then for almost all $x>0$,

$$
\pi(x+\Phi(x))-\pi(x) \sim \frac{\Phi(x)}{\log x} .
$$

We now apply this theorem with the choice $\Phi(x)=x^{1 / 2+\delta}$ to inequality (3.9) and consider the primes $N \leqq p_{m}<p_{m+1}<\cdots<p_{n}<2 N$ in an interval $(N, 2 N)$ with $N$ large. According to Theorem 3.10 the union of the set of intervals $\left(p_{i}, p_{i+1}\right)$ where $p_{i}, p_{i+1}$ satisfy (3.9) and $m \leqq i<n$, form a set of total length $\langle\varepsilon N$ where $\varepsilon>0$ is arbitrarily small. Also the number of indices $i$ for which (3.9) holds is $o(\sqrt{N})$. Thus by (3.8) and (3.9) we have

$$
\begin{aligned}
\frac{c_{n}^{\prime}}{c_{m}^{\prime}} & =\prod_{i=m}^{n-1} \frac{c_{i+1}^{\prime}}{c_{i}^{\prime}}=\prod_{\substack{i=m \\
c^{\prime} i+1^{\prime} i}}^{n-1} \frac{c_{i+1}^{\prime}}{c_{i}^{\prime}}<\frac{N+\varepsilon N}{N}\left(1+o\left(N^{-(+\delta) / 2}\right)\right)^{\sqrt{N}} \\
& <1+2 \varepsilon<2^{2 \varepsilon} .
\end{aligned}
$$

From the monotonicity of $a_{n}$ it now follows that for any $\varepsilon>0$ we have

$$
\left|c_{n}\right|<n^{\varepsilon} \text { for all large } n .
$$

Substituting this inequality in (3.9) we get that $c_{n+1}^{\prime}>c_{n}^{\prime}$ would imply

$$
p_{n+1}>p_{n}+\frac{p_{n}}{c_{n}^{\prime}}-p^{1 / 2+\delta / 4}>p_{n}+\frac{1}{2} p_{n}^{1-\varepsilon}
$$

which is impossible for large $n$ when $\varepsilon<5 / 12$. Thus $\left\{c_{n}^{\prime}\right\}$ becomes nonincreasing for large $n$ and hence constant, $c_{n}^{\prime}=c$, for large $n$. 
This implies $a_{p}>p /(c+1)$ for large primes $p$ and by the monotonicity of $a_{n}$ we get

$$
\frac{a_{n}}{n}>\frac{a_{p}}{2 p}>\frac{1}{4 c}
$$

where $p$ is the largest prime $\leqq n$.

Now consider the successive equations

$$
\begin{aligned}
b_{p} & =c a_{p}-c_{p+1} \\
b_{p+1} & =c_{p+1} a_{p+1}-c_{p+2} .
\end{aligned}
$$

Thus

$$
\begin{gathered}
A \varphi(p+1)+B \sigma(p+1)+O\left(p^{1 / 2-\delta}\right)=c_{p_{+1}} a_{p_{+1}} \\
D p+O\left(p^{1 / 2-\delta}\right)=c a_{p}
\end{gathered}
$$

for all large primes $p$. This leads to

$$
\left|\frac{A}{D} \frac{\varphi(p+1)}{p+1}+\frac{B}{D} \frac{\sigma(p+1)}{p+1}-\frac{c_{p+1}}{c}\right|<p^{-1 / 2},
$$

and hence to the conclusion that the only limit points of the sequence

$$
\left\{\frac{A}{D} \frac{\varphi(p+1)}{p+1}+\frac{B}{D} \frac{\sigma(p+1)}{p+1} \mid p=\text { prime }\right\}
$$

are rational numbers with denominator $c$. To see that this is not the case, consider first the case $B \neq 0$. Then by Dirichlet's theorem about primes in arithmetic progressions we see that $\sigma(p+1) /(p+1)$ is everywhere dense in $(1, \infty)$. Thus we can choose $p$ so that the distance of $B \sigma(p+1) / D(p+1)$ to the nearest fraction with denominator $c$ is greater that $1 /(3 c)$ while at the same time $\sigma(p+1) /(p+1)$ is so large that $|A \varphi(p+1) / D(p+1)|<1 /(3 c)$, contradicting (3.13). If $B=0$ we use the fact that $\varphi(p+1) /(p+1)$ is dense in $(0,1)$ to get the same contradiction.

Finally we must consider the case $A+B=0$. Here we can go through the same argument as before except that we consider the subsequence $b_{2 p}=A \varphi(2 p)+B \sigma(2 p)+C d_{2 p}=2 B p+\left(3 B+C d_{2 p}\right)=2 B p+$ $O\left(p^{1 / 2-\delta}\right)$. As before we get

$$
b_{2 p}=c a_{2 p}-c_{2 p+1} \text { for all large primes } p
$$

which leads to the wrong conclusion that

$$
\left\{\frac{\sigma(2 p+1)}{2 p+1}-\frac{\varphi(2 p+1)}{2 p+1} \mid p=\text { prime }\right\}
$$

has rational numbers with denominator $c$ as its only limit points. 


\section{REFERENCES}

1. P. Erdös, Sur certaines series a valeur irrationelle, Enseignment Math., 4 (1958), 93-100.

2. P. Erdös and E. G. Straus, Some number theoretic results, Pacific J. Math., 36 (1971), 635-646.

3. A. Selberg, On the normal density of primes in small intervals, and the difference between consecutive primes, Arch. Math. Naturvid., 47 (1943), 87-105.

Received April 16, 1974. This work was supported in part under NSF Grant No. GP-28696.

University of California, Los Angeles 


\section{PACIFIC JOURNAL OF MATHEMATICS}

EDITORS

RICHARD ARENS (Managing Editor)

University of California

Los Angeles, Calıfornia 90024

R. A. Beaumont

University of Washington

Seattle, Washington 98105
J. DugundJI

Department of Mathematics

University of Southern California

Los Angeles, California 90007

D. Gilbarg and J. Milgram

Stanford University

Stanford, California 94305

\section{ASSOCIATE EDITORS}
E. F. BECKENBACH
B. H. NeumanN
F. WOLF
K. YOSHIDA

\section{SUPPORTING INSTITUTIONS}

UNIVERSITY OF BRITISH COLUMBIA

CALIFORNIA INSTITUTE OF TECHNOLOGY

UNIVERSITY OF CALIFORNIA

MONTANA STATE UNIVERSITY

UNIVERSITY OF NEVADA

NEW MEXICO STATE UNIVERSITY

OREGON STATE UNIVERSITY

UNIVERSITY OF OREGON

OSAKA UNIVERSITY
UNIVERSITY OF SOUTHERN CALIFORNIA

STANFORD UNIVERSITY

UNIVERSITY OF TOKYO

UNIVERSITY OF UTAH

WASHINGTON STATE UNIVERSITY

UNIVERSITY OF WASHINGTON

AMERICAN MATHEMATICAL SOCIETY NAVAL WEAPONS CENTER 


\section{Pacific Journal of Mathematics}

\section{Vol. 55, No. $1 \quad$ September, 1974}

Robert Lee Anderson, Continuous spectra of a singular symmetric

differential operator on a Hilbert space of vector-valued functions . . . $\quad 1$

Michael James Cambern, The isometries of $L^{p}(X, K) \ldots \ldots \ldots \ldots \ldots . . \ldots$

R. H. Cameron and David Arne Storvick, Two related integrals over spaces of continuous functions ................................

Gary Theodore Chartrand and Albert David Polimeni, Ramsey theory and

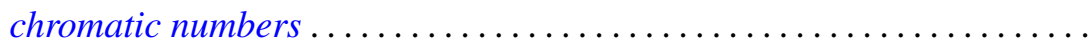

John Deryck De Pree and Harry Scott Klein, Characterization of collectively compact sets of linear operators ...................

John Deryck De Pree and Harry Scott Klein, Semi-groups and collectively compact sets of linear operators ....................... 55

George Epstein and Alfred Horn, Chain based lattices.............. 65

Paul Erdős and Ernst Gabor Straus, On the irrationality of certain series . . 85

Zdeněk Frolík, Measurable uniform spaces................... 93

Stephen Michael Gagola, Jr., Characters fully ramified over a normal

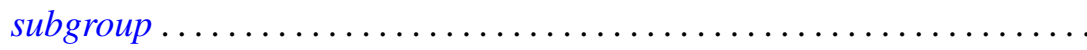

Frank Larkin Gilfeather, Operator valued roots of abelian analytic

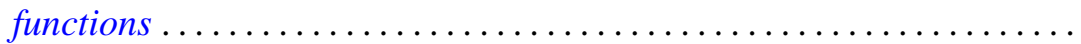

D. S. Goel, A. S. B. Holland, Cyril Nasim and B. N. Sahney, Best approximation by a saturation class of polynomial operators

James Secord Howland, Puiseux series for resonances at an embedded

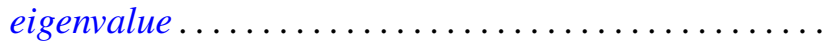

David Jacobson, Linear GCD equations .................

P. H. Karvellas, A note on compact semirings which are multiplicative

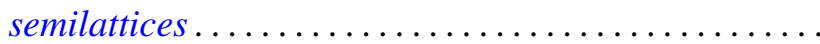

Allan Morton Krall, Stieltjes differential-boundary operators. II . .

D. G. Larman, On the inner aperture and intersections of convex sets

S. N. Mukhopadhyay, On the regularity of the $P^{n}$-integral and its application to summable trigonometric series ....... .

Dwight Webster Read, On $(J, M, m)$-extensions of Boolean algebras ....

David Francis Rearick, Multiplicativity-preserving arithmetic power series.

Indranand Sinha, Characteristic ideals in group algebras

Charles Thomas Tucker, II, Homomorphisms of Riesz spaces . . .

Kunio Yamagata, The exchange property and direct sums of indecomposable injective modules. 